\title{
Wearable integrated piezoelectric film sensor with tensioning, bending, shearing and twisting detection functions for human motion recognition
}

Lu Jin

The University of Manchester

Zhenhong Li

University of Leeds

Zekun Liu

The University of Manchester

Bethany Richardson

The University of Manchester

Yan Zheng

The University of Manchester

Lulu Xu

The University of Manchester

Zhongda Chen

The University of Manchester

Heng Zhai

The University of Manchester

Hongdoo Kim

Kyung Hee University

Qingwen Song

Xi'an Polytechnic University

Pengfei Yue

Xi'an Polytechnic University

Sheng Q. Xie

University of Leeds

Kap Jin Kim

Kyung Hee University

Yi Li ( $\nabla$ henry.yili@manchester.ac.uk)

The University of Manchester 
Keywords: Human-machine Interaction, Biomedical Engineering, Individual Joint Motion, Sensor Deformation, Poly L-lactic Acid Films

Posted Date: March 31st, 2021

DOI: https://doi.org/10.21203/rs.3.rs-290951/v1

License: (c) (1) This work is licensed under a Creative Commons Attribution 4.0 International License. Read Full License

Version of Record: A version of this preprint was published at npj Flexible Electronics on August 17th, 2022. See the published version at https://doi.org/10.1038/s41528-022-00205-4. 


\section{Wearable integrated piezoelectric film sensor with}

2 tensioning, bending, shearing and twisting detection

3 functions for human motion recognition

4 Lu Jin ${ }^{1}$, Zhenhong $\mathrm{Li}^{2}$, Zekun Liu ${ }^{1}$, Bethany Richardson ${ }^{1}$, Yan Zheng ${ }^{1}$, Lulu Xu ${ }^{1}$,

5 Zhongda Chen ${ }^{1}$, Heng Zhai ${ }^{1}$, Hongdoo $\mathrm{Kim}^{3}$, Qingwen Song ${ }^{4}$, Pengfei Yue ${ }^{4}$, Sheng Q.

$6 \mathrm{Xie}^{2 *}, \mathrm{Kap} \mathrm{Jin} \mathrm{Kim}^{3 *}$ and $\mathrm{Yi} \mathrm{Li}^{1,4^{*}}$

$7 \quad{ }^{1}$ Department of Materials, School of Natural Sciences, The University of Manchester,

8 Manchester, M13 9PL, U.K.

$9{ }^{2}$ School of Electronic and Electrical Engineering, University of Leeds, Leeds, LS2 9JT,

10 U.K.

$11{ }^{3}$ Department of Advanced Materials Engineering for Information and Electronics,

12 Kyung Hee University, Yongin, 17104, South Korea

$13{ }^{4}$ College of Textile Science and Engineering, Xi'an Polytechnic University, Xi'an

$14 \quad 710048$, China

$15 *$ Corresponding author:

Yi Li (henry.yili@manchester.ac.uk)

17 Kap Jin Kim (kkim $@$ khu.ac.kr $)$

18 Sheng Q. Xie (‥Q.Xie@leeds.ac.uk) 


\section{Abstract}

Human motion recognition using flexible/stretchable wearable sensors holds great promise for human-machine interaction and biomedical engineering. However, to measure the individual joint motion with multiple degrees of freedom, many sensor networks are normally required and pinpointed onto the targeted area, restricting body movement. This is due to the limitation of current wearable sensors; inferring a sensor deformation based on the sensor's electrical signal is challenging. A new concept of wearable sensor that can recognize how the sensor deforms could radically solve this issue. Here, we report a wearable integrated piezoelectric film sensor (i-PFS) capable of detecting basic deformations. To achieve this, for the first time, we propose a novel design concept of using uniaxially drawn piezoelectric poly L-lactic acid (PLLA) films to engineer unimodal tension, bend, shear, and twist sensors that only respond to their corresponding deformations with the enhanced piezoelectric response and selfshielding function. Based on this, we construct the i-PFS by combining the four unimodal sensors and demonstrate that the i-PFS can detect and differentiate individual deformation modes, such as tensioning, bending, shearing, and twisting. To our best knowledge, the i-PFS is the world's first film-based sensor that identifies the abovementioned deformations. To prove the potential impact of the i-PFS, we design a sleeve and a glove with the i-PFS that can capture various wrist motions and subtle finger movements, respectively. We also develop a virtual textentry interface system using the glove and a deep neural network algorithm with 
a character classification accuracy of $\sim 90 \%$. The i-PFS technology is expected to provide a turning point in developing motion capture systems.

Human motion recognition is of great importance for human-machine interaction ${ }^{1,2}$, entertainment $^{3,4}$, and rehabilitation ${ }^{5,6}$. Several techniques have been developed to measure human motions. One method utilizes the camera-based motion capture system to record a subject's detailed actions, but it requires high computational loads and limits activity range $e^{7,8}$. Another approach is to apply inertial sensors, but the sensors' rigidity confines their application in daily life ${ }^{9,10}$. Alternatively, flexible/stretchable, lightweight wearable sensors, such as piezoelectric sensors, resistive sensors, capacitive sensors, triboelectric sensors, and so forth, provide a promising way for motion monitoring ${ }^{1-16}$. However, the vast majority of human body joints have multiple degrees of freedom ${ }^{17-}$ 19. To identify the complicated individual joint motion precisely, multiple wearable sensors are required and pinpointed onto the desired positions, restricting the naturalness of movements ${ }^{20-25}$. Fundamentally, such inefficiency and impracticality are ascribed to the limitation of existing wearable sensors; gaining information on a sensor deformation from the sensor's electrical signal is a huge challenge. For instance, the wrist has multiple degrees of freedom; it can bend, twist, and even rotate ${ }^{20}$. We can detect an electrical signal from a sensor attached to the wrist joint once deformed. Conversely, it is not easy to infer the wrist deformation (i.e., the sensor deformation) based on the detected electrical signal because any wrist motion generates a signal. It is highly desirable to develop a new type of wearable sensor, which can provide 
feedback on the sensor deformation, to radically solve the above problem.

Hence, we propose a novel concept of a wearable sensor comprising uniaxially drawn piezoelectric poly L-lactic acid (PLLA) films, i.e., an integrated piezoelectric film sensor (i-PFS), which can detect and differentiate four typical deformations, such as tensioning, bending, shearing, and twisting.

\section{Theoretical background}

To develop the i-PFS, we first design four unimodal tension, bend, shear, and twist sensors with the piezoelectric PLLA films, which only respond to tensioning, bending, shearing, and twisting, respectively. The pristine piezoelectric PLLA film only presents a shear piezoelectric coefficient (PC), i.e., $d_{14}$, because of its helical structure ${ }^{26}$. It is usually cut at a certain angle from the crystal orientation (i.e., drawing direction) of the original PLLA film to respond to an external stimulus via modifying its $\mathrm{PC}^{27}$. We, therefore, calculated PCs of four PLLA films with particular cutting angles (CA) such as $45^{\circ},-45^{\circ}, 0^{\circ}$, and $90^{\circ}$ through a mathematical model of the PCs of PLLA film. Of particular interest is that two normal PCs along with the width and length directions, i.e., $d_{12}$ and $d_{13}$, are newly created in the PLLA films with CAs of $45^{\circ}$ and $-45^{\circ}$, named PLLA45 and PLLA-45; simultaneously, the primary shear PC, $d_{14}$, disappears. Besides, the two normal PCs of PLLA45 are opposite to those of PLLA-45. In contrast, the PLLA films with CAs of $0^{\circ}$ and $90^{\circ}$, called PLLA0 and PLLA90, only have the shear PC, $d_{14}$; they are opposite each other as well (see Supplementary Note 1). We then analyzed the four PLLA films' piezoelectric responses when subjected to typical deformations, such as tensioning, bending, shearing, and twisting, via finite element 
simulations using ABAQUS. As shown in the top schematic of Fig. 1a, the deformation directions of tensioning, bending, and shearing (i.e., applied force directions) in simulations cover three axes of a 3-D cartesian system. Notably, a more complex twisting deformation is considered in this work. Thus, these four basic deformation components will introduce any morphologic change to the PLLA film. The PLLA45 and PLLA-45 are sensitive to the tensioning and bending deformations because the average voltage of all nodes of each PLLA film surface $\left(V_{\mathrm{a}}\right)$ is non-zero. Moreover, the generated voltages of each sample are vertically symmetrical, but they are opposite under shearing or twisting and thus offset each other inside (i.e., $V_{\mathrm{a}}=0$ ), not showing a piezoelectric response on the whole. This is attributed to the normal PCs of the PLLA45 and PLLA-45. Similarly, the PLLA0 and PLLA90 are only responsive to the shearing and twisting deformations because of the shear PCs (Fig. 1a and Supplementary Video 1). Overall, each PLLA film is still sensitive to two deformation modes, indicating that it is impossible to determine the exact deformation status with the piezoelectric signal measured from the single-layer PLLA film sensor during deformation. Crucially, we find that the PLLA45 and PLLA-45 generate opposite voltages under the same deformation, and so do PLLA0 and PLLA90.

\section{Design concept and hypothesis of unimodal sensors}

Based on the above findings, we introduce a double-layer PLLA film design concept of unimodal tension, bend, twist, and shear sensors, as illustrated in Fig. 1b,c. We hypothesize that as the unimodal tension sensor consists of two PLLA45s, both receive tensile stress when tensioned, producing the same charges in the inner and outer 
electrodes, respectively; the electric signals of two PLLA45s will be superimposed. Under bending, the upper area of each PLLA45 receives compressive stress, whereas the bottom area is relatively subjected to tensile stress, generating the opposite charges; the signals of two PLLA45s will be canceled out. The charges produced will also be offset inside each PLLA45 under twisting and shearing deformations, not showing signal, as illustrated in Supplementary Fig.2. Conversely, as the unimodal bend sensor is composed of PLLA45 and PLLA-45, the bend sensor's signal will be boosted under bending, but it will be offset when tensioned. To theoretically prove the design concept, the piezoelectric responses of both PLLA films of the unimodal tension, bend, twist, and shear sensors were simulated under different deformations. As long as the unimodal sensors receive their matched deformations, two PLLA films composing each unimodal sensor produce the same piezoelectricity; otherwise, they generate opposite voltages (Fig. 1d), which exactly agrees with our hypothesis.

\section{Unimodal sensors fabrication and performance test}

The piezoelectric property of uniaxially drawn PLLA film is strongly dependent on its crystal orientation and crystallinity, which are dominated by fabrication conditions, especially the drawing ratio $(\mathrm{DR})^{26,28}$. We, therefore, prepared four uniaxially drawn piezoelectric PLLA films with different DRs, such as 3.3, 3.7, 4.0, and 4.5 (Fig. 2a). As shown in Fig. 2b, the crystal orientation improves with increasing the DR because a Debye-Scherrer ring displayed at an initial DR of 3.3 gradually becomes three ellipses at a maximum DR of 4.5 , indicating the highly orientated $\alpha$-crystal structure is formed ${ }^{29}$. This behavior is observed by their melting thermograms as well. After a glass transition 
temperature $\left(\mathrm{T}_{\mathrm{g}}=64^{\circ} \mathrm{C}\right)$, the cold crystallization temperature $\left(\mathrm{T}_{\mathrm{c}}\right)$ of the drawn PLLA films appears at different temperatures, and the lower DR, the higher $\mathrm{T}_{\mathrm{c}}$. This is because

the low chain orientation of the PLLA film requires more energy to form the ordered arrangements and undergoes crystallization at a higher temperature (Fig. 2c). Besides, there is a single melting peak $\left(\mathrm{T}_{\mathrm{m}}\right)$ at around $170^{\circ} \mathrm{C}$, a typical $\alpha$-crystal melting peak ${ }^{26}$, but the $\beta$-crystal melting peak at $\mathrm{T}_{\mathrm{m}}=155^{\circ} \mathrm{C}$ is not observed ${ }^{30}$, further confirming all PLLA films are only compose of $\alpha$-crystal. The crystallinity increases with increasing the DR, and it reaches $60.9 \%$ at a DR of 4.5 (Fig. 2d). As a result, the piezoelectric PLLA film with the DR of 4.5 exhibits the most superior piezoelectric response among them (Fig. 2e). Therefore, the PLLA film with the DR of 4.5 was selected and fabricated into four unimodal tension, bend, twist, and shear sensors following the proposed design concept (Fig. 2f). Notably, as the prepared PLLA film with $\alpha$-crystal (i.e., $10_{3}$ helical structure) does not present a remnant polarization prior to any shear deformation, one cannot determine its resultant polarization direction when it is shear-deformed without a measurement; in other words, which side of the PLLA film produces positive charges or negative charges when deformed. One, however, can hypothesize that the front sides of two PLLA45s and two PLLA0s certainly generate the same charges, whereas those of the PLLA45 and PLLA-45 and the PLLA0 and PLLA90 undoubtedly produce the opposite charges in the identical deformation because they are cut from the same PLLA film sheet. Therefore, to facilitate the process of sensor fabrication, the front sides of the two matched PLLA parts are stuck to each other.

These unimodal sensors were tested one by one under the tensioning, bending, 
twisting, and shearing conditions. Comparing four unimodal sensors under the same deformation condition (i.e., horizontal comparison of Fig. 3a), each sensor exhibits the most significant piezoelectric behavior when received matched stimulus; otherwise, almost no response (P-value $<0.001$, Fig. $3 b$ ). To further evaluate their piezoelectric behavior when subjected to different deformations, the corresponding peak to peak signal amplitudes ( $\left.V_{\mathrm{p}-\mathrm{p}}\right)$ of Fig. $3 \mathrm{~b}$ are normalized (see Method) to compare all signals

161 under the same scale. As expected, all unimodal sensors are sensitive to their corresponding deformations but insensitive to the other conditions (Fig. 3c). Besides, the unimodal sensors can identify the applied force direction (Fig. 3a and Supplementary Fig. 3). We also evaluated the stability and repeatability of the unimodal sensors. The stable and continuous piezoelectric response during 1000 cycles and the reproduced piezoelectric signals after each day without significant variation demonstrate signal stability and repeatability (Supplementary Fig. 4). The unimodal tension sensor exhibits a relatively stronger piezoelectric noise signal than the other sensors under unmatched deformations (Fig. 3c). This is ascribed to the mismatching of two PLLA45s composing the tension sensor. The operating mechanism of these sensors is amplifying their signals when subjected to the corresponding stimuli but

172 offsetting under unmatched deformations. The latter is a key for the unimodal sensors, 173 and it is much more difficult to be achieved than the former. This is because a slight 174 discordance in piezoelectric and physical properties of two PLLA films, such as CA, 175 drawing direction, size, and thickness, could result in a non-negligible noise signal even under the non-corresponding stimuli. For instance, a slight deviation on the CA of the 
PLLA45 can cause the residual shear piezoelectric coefficient, $d_{14}$, in the PLLA45, which acts to shearing and twisting deformations. The size or thickness difference can also lead to the uneven force receiving between two PLLA45s. To minimize such discrepancies, the two PLLA parts of each unimodal sensor should be cut from the same area of the original PLLA film, and the closer the two parts, the better the performance. Another difficulty in the unimodal sensor fabrication is finding the exact uniaxial drawing direction of the transparent PLLA film because an incorrect drawing direction could lead to an inevitable error in the CA.

Fig. 3d compares the piezoelectric responses of the four unimodal sensors and conventional PLLA film (single layer) sensors following the above conditions. Notably, all unimodal sensors exhibit much higher piezoelectric responses than the PLLA sensors under corresponding deformations, but their increased levels are different. Under tensioning and shearing, the $V_{\mathrm{p}-\mathrm{p}}$ of the tension and shear sensors almost double compared to the single PLLA film sensors. It is reasonable because the tension and shear sensors consist of two PLLA films, producing coherent piezoelectric signals under the corresponding deformation. For the bend sensor, its $V_{\mathrm{p}-\mathrm{p}}$ is twenty times stronger than that of the PLLA45 sensor. Under the same bending curvature, the bend sensor's two PLLA45 films should be subjected to more massive bending stress than the PLLA45 sensor because of the thicker thickness. Thus it does not merely generate a doubled signal but produces a much stronger signal than the PLLA45 sensor. Similarly, the twist sensor presents a boosted piezoelectric behavior compared with the PLLA0 sensor; significantly, it shows about seventy times stronger signal than the PLLA0 
sensor at a twisted angle of $30^{\circ}$. To further confirm this, we compared the $V_{\text {p-p }}$ of two sensors at twisted angles of $20^{\circ}$ and $40^{\circ}$, which all show consistent results 201 (Supplementary Fig. 5).

202 Owing to the capacitive feature of piezoelectric sensors, including the conventional piezoelectric PLLA sensor, they are quite susceptible to electromagnetic (EM) interference (e.g., $50 \mathrm{~Hz}$ EM noise originated from the household electricity) and motion artifact in a real-world setting ${ }^{31}$. It is easy to eliminate the $50 \mathrm{~Hz}$ EM noise using an appropriate electronic filter system because of the regular frequency. Conversely, most motion artifact caused by the human body has an irregular frequency; it could be challenging to be removed using a particular electronic filter system. Therefore, the 209 piezoelectric sensors themselves should have a proper EM shielding function to apply 210 for wearables. Since the outer electrode of each unimodal sensor covers the inner 211 electrode completely and is connected to a metallic shield part of a coaxial cable (Fig. 212 1c), it can serve as an EM shielding layer. In contrast to the unshielded PLLA45 sensor, 213 the self-shielded unimodal tension sensor exhibits excellent noise-screening 214 performance against the $50 \mathrm{~Hz}$ EM noise and motion artifact (Fig. 3e and 215 Supplementary Video 2). Besides, the PLLA film is quite vulnerable to moisture/water 216 because of the biodegradable nature ${ }^{28,32}$. The outer electrode covers all outside surfaces 217 of the sensors, seamlessly protecting the PLLA films from moisture/water. Nevertheless, 218 for the applications of biodegradable sensors, their durability could also be adjustable 219 by changing the outer electrode's property, e.g., biodegradable materials-based 220 electrodes ${ }^{5,28,33}$. 
We then fabricate the i-PFS by stacking four unimodal sensors together, as shown in Fig. 3f. The i-PFS was tensioned, bent, twisted, and sheared using the relevant machines. The result proves that the i-PFS has enough capability to detect and differentiate tensioning, bending, twisting, and shearing deformations, respectively (Fig. $3 \mathrm{f}$ and Supplementary Fig. 6). More importantly, this demonstrates that the four unimodal sensors composing the i-PFS can work together without evident interference. This is mainly because the individual unimodal sensor is shielded by each outer electrode, not affecting each other. The i-PFS was also deformed by hand to investigate the practicability. As expected, the i-PFS can discriminate imposed motions separately, as shown in Supplementary Fig. 7 and Supplementary Video 3.

\section{Motion recognition applications}

Unlike most piezoelectrics, the piezoelectric PLLA film has no pyroelectricity, which means its signal is not influenced by temperature fluctuation ${ }^{27}$, presenting the unique advantage of the i-PFS in real-life applications. To demonstrate the potential of the iPFS in human motion recognition applications, we fabricated a sleeve with the i-PFS (i-Sleeve for short) to measure various wrist motions. Since the four unimodal sensors composing the i-PFS independently respond to their corresponding deformations

239 (tensioning, bending, shearing, and twisting), the i-Sleeve can differentiate complex wrist motions involving multiple degrees of freedom (e.g., extension/flexion, radial/ulnar deviation, pronation/supination) as shown in Supplementary Fig. 8 and Supplementary Video 4. More importantly, a complicated wrist turning action 
comprising three bending, twisting, and shearing deformation components is simultaneously detected by the bend, twist, and shear sensor channels, respectively. This further implies that the i-PFS could decouple complex human motions. Supplementary Table 1 summarizes relevant works on wrist motion capture. The iSleeve is unique as it does not require multiple sensors that are distributed to the desired area. It utilizes a single i-PFS to further identify wrist turning action that is superior to conventional approaches, showing the great efficiency of the i-PFS.

Furthermore, the i-PFS was integrated into an index finger of a glove (i-Glove for short) to evaluate its performance in subtle motion detection (the bottom inset of Fig. 4a), i.e., index finger movements. Due to the limited surface area available on a finger, it is challenging to attach multiple sensors. Therefore, most previous studies focused on tracking the fingers' flexion and extension motion (Supplementary Table 2). It is still an open issue to capture index finger movements involving multiple degrees of freedom with wearable sensors. The i-PFS of the i-Glove can discriminate various finger modes, such as bending up and down, shearing left and right, turning clockwise and anticlockwise, and flexion and extension (Fig. 4b). Besides, the i-PFS can distinguish the finger movements' directions.

Based on this, we designed a virtual text-entry interface system using the i-Glove in conjunction with a convolutional neural network $(\mathrm{CNN})$ algorithm for a finger-airwriting application, which detects finger movements with the i-PFS and transforms them into corresponding characters (Fig. 4a,c and Supplementary Video 5). We chose 13 characters (i.e., "U”, “O”, “M”, “0”, “1”, “2”, “3”, “4”, “5”, “6”, “7”, “8”, “9”) as 
target classes. One participant was invited to create the data source for the finger-airwriting (see Method). As the character writing habit influences the output signal, the writing style of each character was defined before collecting data to eliminate its effect. For instance, we instructed the participant to write the letter "O" in clockwise and the number " 0 " in anti-clockwise to distinguish them. Supplementary Fig. 9 displays the output data of 13 characters of three trials, which shows good signal reproducibility. We then adopted a LeNet-5 based CNN architecture consisting of two convolutional layers for character classification (Supplementary Fig. 10). The classification result shows that the accuracy using four channels can reach $89.7 \%$ (Fig. 4d). We also examined the classification accuracies of individual sensor channels, two and threechannel combinations. The results show that the testing accuracies increase with increasing the channel numbers; the mean classification accuracy can reach $85 \%$ when using any three sensor channels (Supplementary Fig. 11-13). Several groups have exploited the vision-based system ${ }^{7,8}$, the inertial sensors ${ }^{34}$, and their combination ${ }^{35}$ for air-writing implementation. Unlike these approaches, our finger-air-writing is realized merely using the wearable i-PFS to detect the subtle metacarpophalangeal joint movement of the index finger, demonstrating the performance of the i-PFS in minute motion monitoring.

\section{Conclusion}

In summary, we present a new type of wearable sensor, i.e., i-PFS, capable of identifying tensioning, bending, twisting, and shearing deformations and demonstrate the potential impact of the i-PFS in motion recognition systems. To realize this, four 
unimodal sensors for the i-PFS are designed and fabricated by sticking two uniaxially drawn piezoelectric PLLA films with different CAs, endowing the sensors with the

289 functions of not only detecting and differentiating unimodal deformations but also the 290 self-shielding ability. These unimodal sensors and their combinations can be utilized as 291 various film-based force, strain, and deformation sensors with 1, 2, or 3-axial sensitivity depending on the purpose. Significantly, the sensors will be incredibly beneficial in the 293 applications of implantable and biodegradable sensors because of the excellent 294 biocompatibility and biodegradability of the PLLA. We also expect that the novel design principle of unimodal piezoelectric sensors and i-PFS proposed here can be applied to other piezoelectrics, making them more powerful and smarter. 


\section{Methods}

Piezoelectric response simulations of four uniaxially drawn piezoelectric PLLA

films with various cutting angles under tensioning, bending, shearing, and twisting

Parameters used for the piezoelectric response simulations are as follows: a PLLA piezoelectric coefficient $\left(d_{14}\right)$ of $10 \times 10^{-12} \mathrm{C} / \mathrm{N}$, a PLLA density of $1250 \mathrm{~kg} / \mathrm{m}^{3}$, a PLLA Young modulus of $4 \times 10^{9} \mathrm{~Pa}$, a PLLA Poisson's ratio of 0.36 , and all parameters are estimated values based on the reference ${ }^{36}$. The dimension of the PLLA films (Length $\times$ Width $\times$ Thickness) is $2 \times 1 \times 0.01 \mathrm{~cm}$. The applied forces for tensioning, bending, and shearing deformations are $1 \times 10^{4} \mathrm{~Pa}, 1 \times 10^{1} \mathrm{~Pa}$, and $1 \times 10^{3} \mathrm{~Pa}$, respectively. For twisting deformation, the PLLA film is vertically divided into two parts, and one part applies $1 \times 10^{3} \mathrm{~Pa}$ and the other part $-1 \times 10^{3} \mathrm{~Pa}$.

\section{PLLA chips for uniaxially drawn piezoelectric PLLA film preparation}

Ingeo biopolymer 4032D $\left(\mathrm{M}_{\mathrm{w}} \approx 195000\right.$, NatureWorks, USA $)$ with $98 \%$ L-isomer and

$2 \%$ D-isomer was used for uniaxially drawn piezoelectric PLLA film preparation.

Output voltage measurement under tensioning, bending, shearing, and twisting deformations

The output voltages were acquired in the voltage mode of a Piezo Film Lab Amplifier (Measurement Specialties, Inc., USA) in a condition of an input impedance of $10 \mathrm{M} \Omega$, a band-pass filter range of $0.1-10 \mathrm{~Hz}$, and a gain value of $0 \mathrm{~dB}$ at $100 \mathrm{~Hz}$ of the sampling rate. For the shielding performance test, the input impedance and the band-pass filter range of the Piezo Film Lab Amplifier increase to $1 \mathrm{G} \Omega$ and $0.1-1000 \mathrm{~Hz}$, respectively. 
Tensioning deformation was realized using an electromechanical universal testing system (Instron, a strain of $1 \%$ ), and the other deformations were performed using a bending machine (a bending angle of $21.8^{\circ}$ ), a twisting machine (a twisted angle of $30^{\circ}$ ), and a shearing machine (a shear strain of 0.05 ) with a frequency of $0.5 \mathrm{~Hz}$, as illustrated in Supplementary Fig. 14.

\section{Characterizations of uniaxially drawn piezoelectric PLLA films}

To determine the crystal orientation and crystallinity of the uniaxially drawn piezoelectric PLLA film, two-dimensional wide-angle x-ray diffraction (2D-WAXD) photograph and the corresponding 1D-WAXD spectrum were obtained in reflection mode using a Rigaku SmartLab 3K diffractometer with a $\mathrm{Cu} \mathrm{K} \alpha(\lambda=1.54 \AA)$ radiation source ranging from $2 \theta=4^{\circ}$ to $40^{\circ}$. Crystallinity percentages of the PLLA films were quantified from the curve deconvolutions of their corresponding 1D-WAXD spectrums. The melting thermograms were measured utilizing a differential scanning calorimeter (DSC Q2000, TA Instruments, USA) at a heating rate of $10{ }^{\circ} \mathrm{C} / \mathrm{min}$ from 40 to $190{ }^{\circ} \mathrm{C}$ at nitrogen atmosphere.

\section{Unimodal sensors' signals normalization}

347 To compare each unimodal sensor's piezoelectric response under different deformation conditions, its noise signal under unmatched conditions should be considered because it increases with increasing non-corresponding deformation degree/intensity. For example, under tensioning deformation, the signals recorded from the bend, twist, and shear sensors are regarded as noises; these noises certainly increase with increasing the tensioning intensity that is directly reflected by the tension sensor, as shown in Fig. 3b. 
In other words, the noises increase with increasing the signal of the tension sensor. This

causes big trouble in comparing each sensor's exact piezoelectric response under different deformations and their noise levels. To solve this problem, all $V_{\mathrm{p}-\mathrm{p}}$ of the sensors under each deformation condition are normalized by dividing the $V_{\mathrm{p}-\mathrm{p}}$ of the matched sensor under the same deformation (below equations) to place all signals on the same scale $(0 \sim 1)$ as summarized in Fig. $3 \mathrm{c}$.

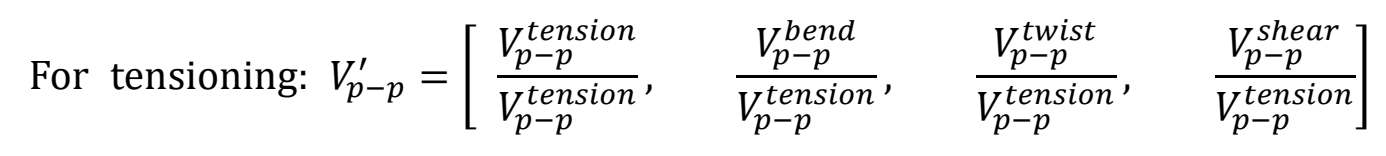

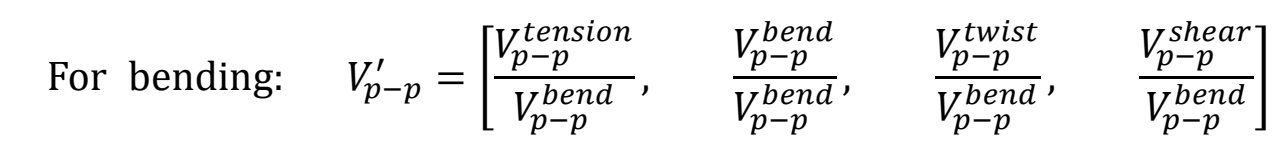

For twisting: $\quad V_{p-p}^{\prime}=\left[\frac{V_{p-p}^{\text {tension }}}{V_{p-p}^{\text {twist }}}, \quad \frac{V_{p-p}^{\text {bend }}}{V_{p-p}^{\text {twist }}}, \quad \frac{V_{p-p}^{\text {twist }}}{V_{p-p}^{\text {twist }}}, \quad \frac{V_{p-p}^{\text {shear }}}{V_{p-p}^{\text {twist }}}\right]$

$$
\text { For shearing: } \quad V_{p-p}^{\prime}=\left[\frac{V_{p-p}^{\text {tension }}}{V_{p-p}^{\text {shear }}}, \quad \frac{V_{p-p}^{\text {bend }}}{V_{p-p}^{\text {shear }}}, \quad \frac{V_{p-p}^{\text {twist }}}{V_{p-p}^{\text {shear }}}, \quad \frac{V_{p-p}^{\text {shear }}}{V_{p-p}^{\text {shear }}}\right]
$$

where $V_{p-p}^{\prime}$ is the normalized $V_{\mathrm{p}-\mathrm{p}}, V_{p-p}^{\text {tension }}$ is the $V_{\mathrm{p}-\mathrm{p}}$ of the tension sensor under each deformation, $V_{p-p}^{\text {bend }}$ is the $V_{\mathrm{p}-\mathrm{p}}$ of the bend sensor, $V_{p-p}^{\text {twist }}$ is the $V_{\mathrm{p}-\mathrm{p}}$ of the twist sensor, and $V_{p-p}^{\text {shear }}$ is the $V_{\mathrm{p}-\mathrm{p}}$ of the shear sensor.

\section{Signal collection and process of the finger-air-writing}

The participant is asked to perform 100 trials of air-writing wearing the i-Glove. In each test, the participant continuously writes the 13 characters using the index finger, and the writing time for each character is about $2 \mathrm{~s}$. The output data of i-PFS from four channels are recorded at $100 \mathrm{~Hz}$. The collected multi-channel data are then filtered digitally using a sixth-order low-pass Butterworth filter with a $10 \mathrm{~Hz}$ cut-off frequency and is segmented using a $1 \mathrm{~s}$ sliding window with $0.2 \mathrm{~s}$ increment. Since the spectrum 
373 of output data from i-PFS is observed to be less noisy and more distinguishable than 374 the output data in the temporal domain, each segmented data channel is applied for fast 375 Fourier transform (FFT). The transformed data is split into $80 \%$ and $20 \%$ and used as 376 a training set and test set of CNN, respectively. 


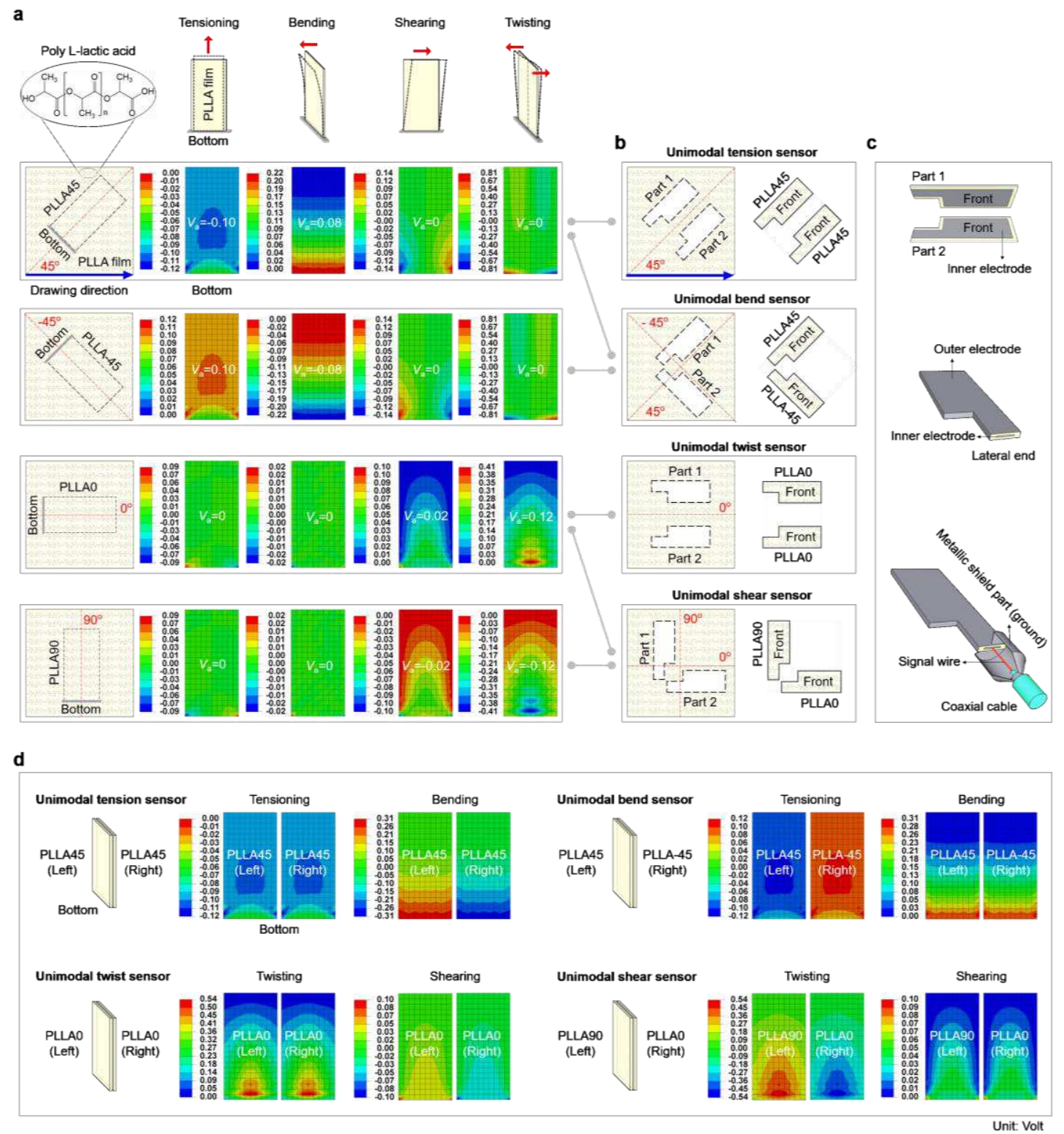

Fig. 1. Design concept of unimodal tension, bend, twist, and shear sensors. a, Top

schematics show the applied forces and their directions (red arrows) for tensioning, bending, shearing, and twisting deformations in finite element simulations. The bottom panels show the piezoelectric response simulations of the uniaxially drawn piezoelectric PLLA films with different cutting angles under tensioning, bending,

397 shearing, and twisting deformations. The first panel shows the uniaxially drawn piezoelectric PLLA film with a cutting angle of $45^{\circ}$ (PLLA45 for short) and its cutting schematic (first graph) with a cutting shape (black dash line) and a cutting angle (red 
dash line), and a PLLA molecular formula shown in the circle. The drawing direction (blue arrow) of the PLLA film is the same as the PLLA molecular chain's direction. The average responding voltage of all nodes of the PLLA film surface $\left(V_{\mathrm{a}}\right)$ is shown in the middle, and the PLLA film bottom is fixed when it is subject to tensioning, bending, shearing, and twisting forces. The same illustrative schemes are applied for PLLA-45, PLLA0, and PLLA90. b, Left schematics show the cutting angles (red dash lines) and cutting shapes (black dash line) of two parts from an original piezoelectric PLLA film for the unimodal tension, bend, twist, and shear sensors, respectively, and the right schematics show the corresponding parts after cutting out; c, Top schematic shows the patterns of inner electrodes (gray areas), and the front sides (i.e., inner electrodes) of two parts of each sensor are stuck together; the middle one shows the exposed area of each assembled sensor is coated with silver paste as an outer electrode, except the lateral end of the sensor; the bottom one shows the inner electrodes are connected to a signal wire, and the outer electrode is connected to a metallic shield part (ground) of a coaxial cable. d, Piezoelectric response simulations of two PLLA films composing each unimodal sensor under tensioning, bending, shearing, and twisting deformations to prove the unimodal sensors' hypothesis. The first panel shows the piezoelectric response of two PLLA45s of the unimodal tension sensor under tensioning and bending. The piezoelectric responses of the exposed areas of two PLLA45s are shown in simulation; in other words, the displayed voltages are on outer electrodes of the unimodal tension sensor. The generated voltages of the two PLLA45s are superimposed when tensioned, but they are offset under bending. The unimodal tension sensor under 
423 deformations, as demonstrated in Fig. 1a. The same illustrative schemes are applied for 424 the unimodal bend sensor under tensioning and bending, the unimodal twist sensor 425 under twisting and shearing, and the unimodal shear sensor under twisting and shearing. 426 Note that all parameters and applied forces used in these simulations (Fig. 1d) are the 427 same as the previous piezoelectric PLLA film simulations (Fig. 1a).

428

429

430

431

432

433

434

435

436

437

438

439 


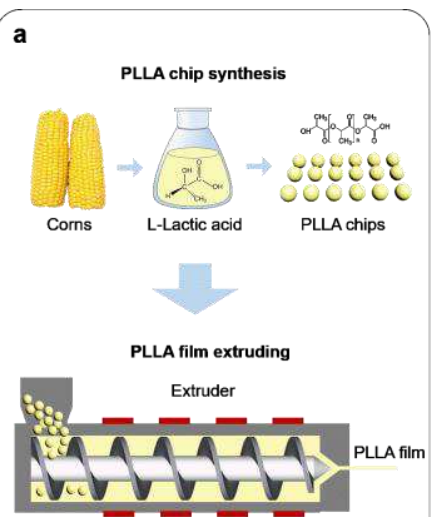

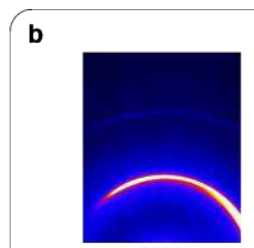

DR3.3

c
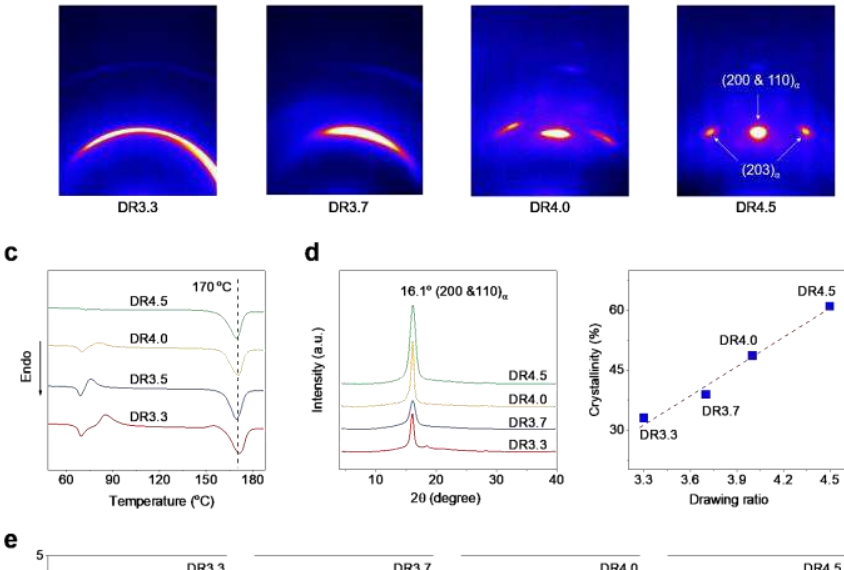

DR3.7

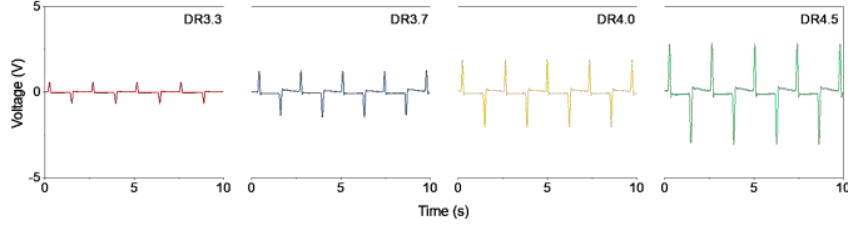

$\mathbf{f}$
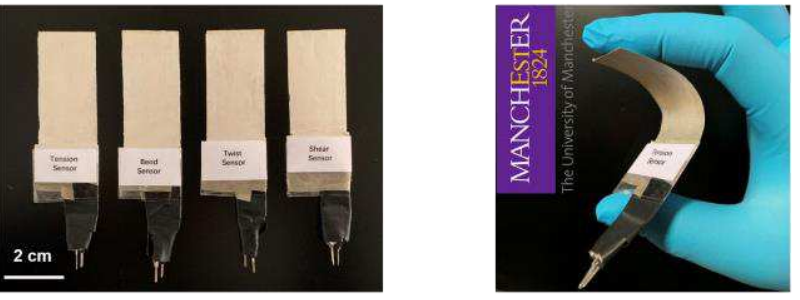

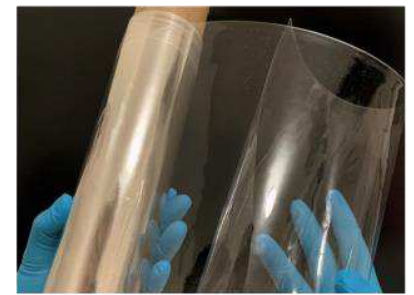

Fig. 2. Preparation and characterization of uniaxially drawn piezoelectric PLLA

films. a, Schematic of uniaxially drawn piezoelectric PLLA films preparation process.

The first photograph shows the PLLA chip synthesis, which is polymerized from Llactic acid that is obtained from the fermentation of renewable and biodegradable plant sources such as corns ${ }^{37}$. In this work, we directly purchased the PLLA chips from NatureWorks, USA. The second photograph shows the PLLA film extruding. The PLLA chips are extruded into PLLA film at $225^{\circ} \mathrm{C}$ using an extruder after dehumidifying at $120^{\circ} \mathrm{C}$ under a vacuum for eight hours. The third photograph shows that PLLA film is stretched at different drawing ratios (DR) of 3.3, 3.7, 4.0, and 4.5 at 450 $70{ }^{\circ} \mathrm{C}$, and each sample names DR3.3, DR3.7, DR4.0, and DR4.5, respectively. The 
451 bottom photograph shows a transparent piezoelectric PLLA film (DR4.5). b, Two452 dimensional wide-angle x-ray diffraction (2D-WAXD) photographs of four PLLA film 453 samples for crystal orientation analysis; c, Differential scanning calorimeter (DSC) 454 melting thermograms of the PLLA films with different DRs for crystal form 455 determination. d, 1D-WAXD spectrum of the PLLA films for crystal form 456 determination (left) and their crystallinity (right). e, Comparison of piezoelectric 457 response of four PLLA film samples under tensioning deformation. The dimension 458 (Length $\times$ Width) of each sensor is $4 \times 2 \mathrm{~cm}$, and every PLLA film is cut at $45^{\circ}$ from 459 the drawing direction. f, Photographs of four unimodal sensors (left) and the sensor 460 flexibility demonstration (right).

461

462 463 464 465 466 467 468 

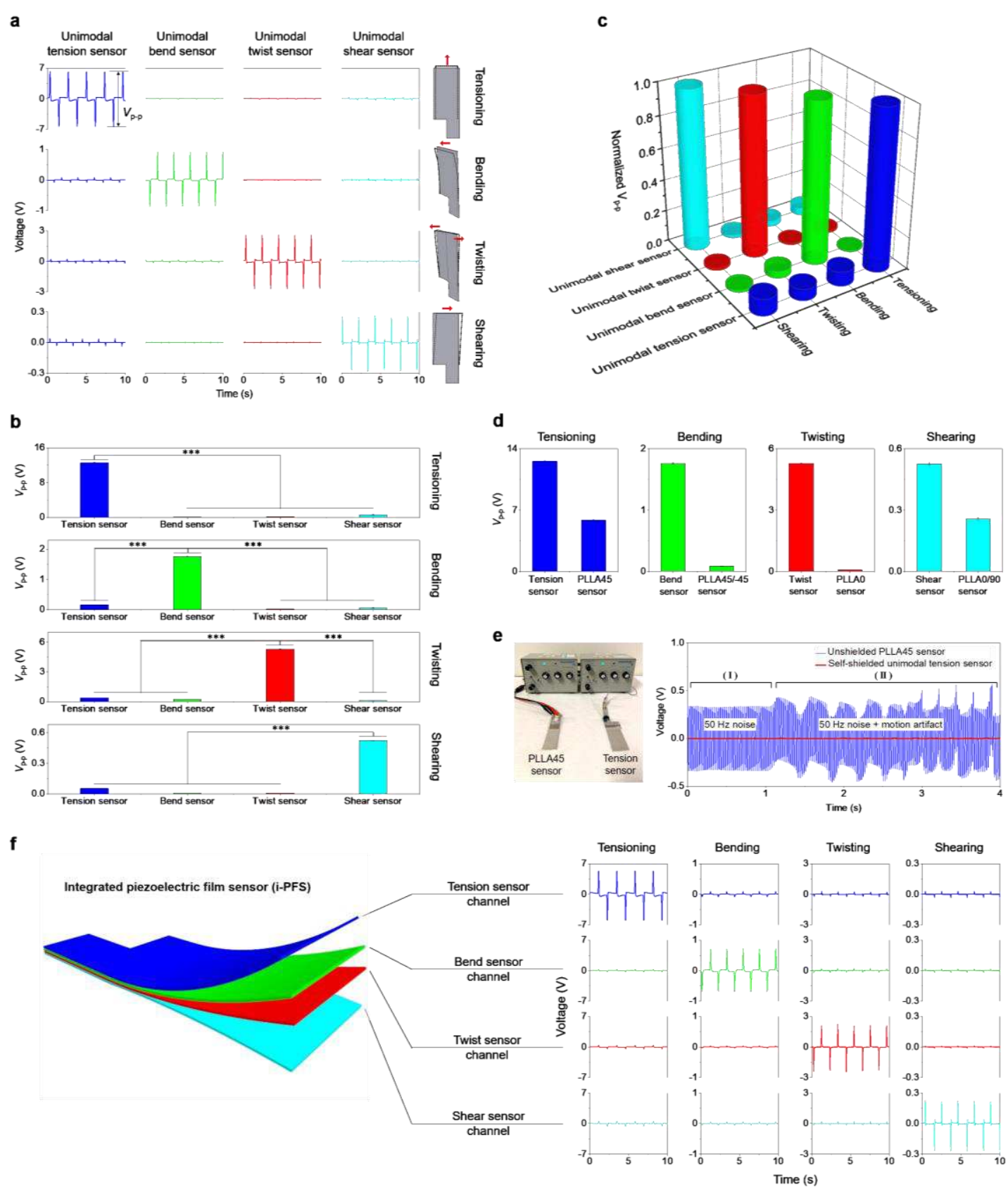

Fig. 3. Performance evaluation of four unimodal sensors and integrated piezoelectric film sensor (i-PFS). a, Piezoelectric responses of each unimodal sensor

472 under the same tensioning, bending, twisting, and shearing conditions. Schematics of

473 unimodal tension, bend, twist, and shear sensors with their corresponding deformations

474 are shown in right insets. b, Comparison of the corresponding peak to peak signal amplitudes $\left(V_{\mathrm{p}-\mathrm{p}}\right)$ of Fig. 3a $(* * *$ indicating P-value $<0.001)$. c, Normalized $V_{\mathrm{p}-\mathrm{p}}$ of 
476 unimodal tension, bend, twist, and shear sensors under tensioning, bending, twisting, 477 and shearing deformation conditions. d, Comparison of the piezoelectric response 478 between the four unimodal sensors and the conventional single-layer PLLA sensors 479 under the above conditions. e, The left photograph shows an experimental setup for the 480 shielding performance test. The right figure shows the shielding performance of the 481 unshielded PLLA45 sensor and the self-shielded unimodal tension sensor (I) under the 482 static condition and (II) when walking/running next to two sensors. f, Schematic of the 483 i-PFS comprising unimodal tension, bend, twist, and shear sensors (left). The right 484 panels show the piezoelectric response of the i-PFS under the same tensioning, bending, 485 twisting, and shearing conditions. All conditions are the same as those used for 486 individual unimodal sensor (Fig. 3a). 
a

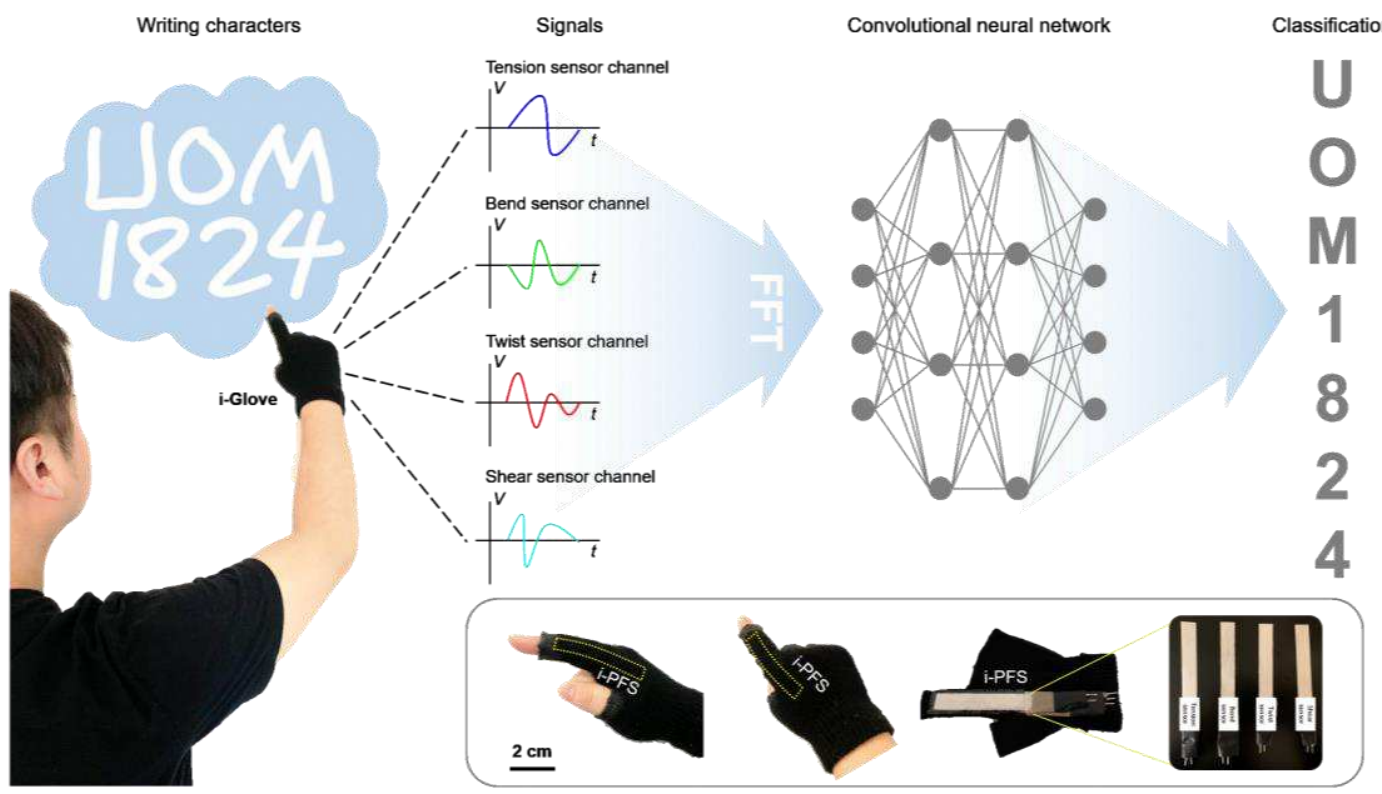

b
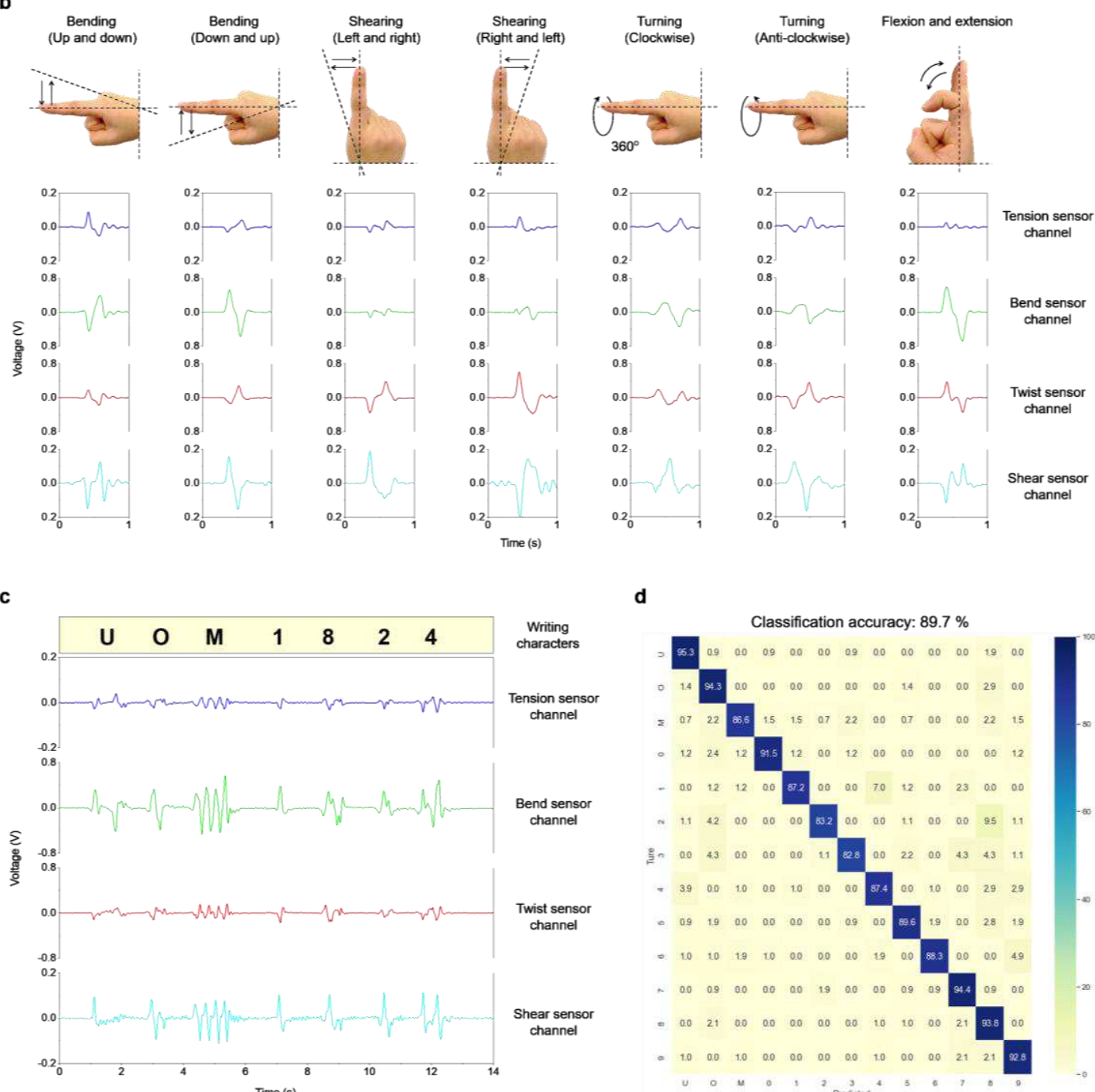

d

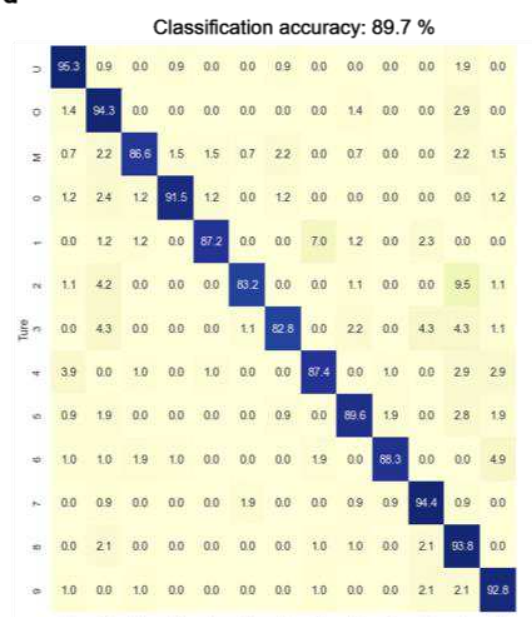

Fig. 4. Demonstration of an i-PFS integrated glove (i-Glove) for finger-air-writing 
application. a, Schematic illustration of a finger-air-writing application. A participant

497 wearing the i-Glove writes some characters, e.g., “U”, “O”, “M”, “1”, “8”, “2”, “4”, in

498 the free space using his index finger. The four unimodal sensors of the i-PFS collect the

499 corresponding signal of each character. The acquired signals are input into a pre-trained

500 convolutional neural network (CNN) program after fast fourier transform (FFT). Finally,

501 the program classifies the characters based on the signals. The bottom first two

502 photographs show the i-PFS positions in the i-Glove at the side and top views. The third

503 photograph shows the inside view of the i-Glove, in which the i-PFS is inserted into a

504 transparent pocket of the index finger. The last photograph shows four unimodal sensors

505 of the i-PFS. b, Index finger motion classification. Top photographs show index finger

506 motions (bending, shearing, turning, and flexion and extension), and the bottom panels

507 show corresponding raw output voltages from four unimodal sensor channels of the i-

508 PFS. c, Top panel shows writing characters, and the bottom panel shows corresponding

509 raw output voltages of four unimodal sensor channels of the i- PFS. d, Confusion matrix

510 for the thirteen characters' classification accuracy and its mean accuracy (top) when

511 using four unimodal sensor channels of the i-PFS. 


\section{References}

519 1. Sundaram, S. et al. Learning the signatures of the human grasp using a scalable $520 \quad$ tactile glove. Nature 569, 698-702 (2019).

521 2. Dong, W. et al. Soft human-machine interfaces: design, sensing and stimulation. Int. J. Intell. Robot. Appl. 2, 313-338 (2018).

3. Song, K. et al. Pneumatic actuator and flexible piezoelectric sensor for soft virtual reality glove system. Sci. Rep. 9, 1-8 (2019).

4. Kim, K. K. et al. A deep-learned skin sensor decoding the epicentral human motions. Nat. Commun. 11, 1-8 (2020).

5. Boutry, C. M. et al. A stretchable and biodegradable strain and pressure sensor for orthopaedic application. Nat. Electron. 1, 314-321 (2018).

6. Sun, T. et al. Decoding of facial strains via conformable piezoelectric interfaces. Nat. Biomed. Eng. 4, 954-972 (2020).

7. Misra, S., Singha, J. \& Laskar, R. H. Vision-based hand gesture recognition of alphabets, numbers, arithmetic operators and ASCII characters in order to

8. Mukherjee, S., Ahmed, S. A., Dogra, D. P., Kar, S. \& Roy, P. P. Fingertip develop a virtual text-entry interface system. Neural Comput. Appl. 29, 117-

9. Homayounfar, S. Z. \& Andrew, T. L. Wearable Sensors for Monitoring Human 
541 10. Stenlund, T. C. et al. Inter- and intra-tester reliability when measuring seated spinal postures with inertial sensors. Int. J. Ind. Ergon. 44, 732-738 (2014).

543 11. Dagdeviren, C. et al. Recent progress in flexible and stretchable piezoelectric devices for mechanical energy harvesting, sensing and actuation. Extrem. Mech. Lett. 9, 269-281 (2016).

12. Chorsi, M. T. et al. Piezoelectric Biomaterials for Sensors and Actuators. Adv. Mater. 31, 1-15 (2019).

13. Dai, Y., Hu, H., Wang, M., Xu, J. \& Wang, S. Stretchable transistors and functional circuits for human-integrated electronics. Nat. Electron. 4, 17-29

14. Wang, W. et al. Strain-insensitive intrinsically stretchable transistors and

16. Moin, A. et al. A wearable biosensing system with in-sensor adaptive machine

15. Chen, L., Fu, J., Wu, Y., Li, H. \& Zheng, B. Hand Gesture Recognition Using Compact CNN via Surface Electromyography Signals. Sensors (Switzerland) 20, 672 (2020).

18. Burke, B. Y. D., Gandevia, S. C. \& Macefield, G. Responses to passive 
movement of receptors in joint, skin and muscle of the human hand. J. Physiol.

563

564

565

566

567

568

569

570

571

572

573

574

575

576

577

578

579

580

581

582

583 402, 347-361 (1988).

19. Tu, Y., Liu, L., Li, M., Chen, P. \& Mao, Y. A review of human motion monitoring methods using wearable sensors. Int. J. Online Eng. 14, 168-179 (2018).

20. Huang, B. et al. Wearable stretch sensors for motion measurement of the wrist joint based on dielectric elastomers. Sensors (Switzerland) 17, (2017).

21. Cha, Y., Seo, J., Kim, J. S. \& Park, J. M. Human-computer interface glove using flexible piezoelectric sensors. Smart Mater. Struct. 26, 5 (2017).

22. Araromi, O. A. et al. Ultra-sensitive and resilient compliant strain gauges for soft machines. Nature 587, 219-224 (2020).

23. Souri, H. et al. Wearable and Stretchable Strain Sensors: Materials, Sensing Mechanisms, and Applications. Adv. Intell. Syst. 2, 2000039 (2020).

24. Leber, A., Cholst, B., Sandt, J., Vogel, N. \& Kolle, M. Stretchable Thermoplastic Elastomer Optical Fibers for Sensing of Extreme Deformations. Adv. Funct. Mater. 29, 1-8 (2019).

25. Chander, H. et al. Wearable stretch sensors for human movement monitoring and fall detection in ergonomics. Int. J. Environ. Res. Public Health 17, 1-18 (2020).

26. Fukada, E. Recent developments of polar piezoelectric polymers. IEEE Trans. Dielectr. Electr. Insul. 13, 1110-1119 (2006).

27. Ando, M., Kawamura, H., Kageyama, K. \& Tajitsu, Y. Film sensor device 

4 (2012).

28. Curry, E. J. et al. Biodegradable Piezoelectric Force Sensor. Proc. Natl. Acad. Sci. 115, 909-914 (2018).

29. $\mathrm{Ru}$, J. F. et al. Dominant $\beta$-form of poly(l -lactic acid) obtained directly from melt under shear and pressure fields. Macromolecules 49, 3826-3837 (2016).

30. Yin, H. M. et al. Effects of extrusion draw ratio on the morphology, structure and mechanical properties of poly(L-lactic acid) fabricated using solid state ram extrusion. $R S C A d v$. 5, 69016-69023 (2015).

31. Atalay, A. et al. Batch Fabrication of Customizable Silicone-Textile Composite Capacitive Strain Sensors for Human Motion Tracking. Adv. Mater. Technol. 2, $1-8(2017)$.

32. Curry, E. J. et al. Biodegradable nanofiber-based piezoelectric transducer. Proc. Natl. Acad. Sci. U. S. A. 117, 214-220 (2020).

33. Someya, T., Bao, Z. \& Malliaras, G. G. The rise of plastic bioelectronics. Nature 540, 379-385 (2016).

34. Amma, C., Georgi, M. \& Schultz, T. Airwriting: Hands-free mobile text input by spotting and continuous recognition of $3 \mathrm{~d}$-space handwriting with inertial sensors. Proc. - Int. Symp. Wearable Comput. ISWC 52-59 (2012). doi:10.1109/ISWC.2012.21

35. Chen, M., AlRegib, G. \& Juang, B. H. Air-Writing Recognition - Part I: Modeling and Recognition of Characters, Words, and Connecting Motions. 
607

36. Smith, M., Calahorra, Y., Jing, Q. \& Kar-Narayan, S. Direct observation of shear piezoelectricity in poly-l-lactic acid nanowires. APL Mater. 5, (2017).

37. Muller, J., González-Martínez, C. \& Chiralt, A. Combination Of Poly(lactic) acid and starch for biodegradable food packaging. Materials (Basel). 10, 1-22 (2017).

\section{Acknowledgements}

This work is financially supported by the EU Horizon 2020 through project ETEXWELD-H2020-MSCA-RISE-2014 (Grant No. 644268) and the University of Manchester through the UMRI project "Graphene-Smart Textiles E-Healthcare Network" (Grant No. AA14512).

\section{Author contributions}

The work was conceived and designed by L.J., Y.L., and K.J.K. L.J. performed the mathematical model and the piezoelectric response simulations and discussed them with Y.L. and K.J.K. H.K. and K.J.K. prepared the uniaxially drawn piezoelectric PLLA film. L.J. and L.X. supervised sensor fabrication. Z.L. and C.D. ran the piezoelectric response test. Y.Z. assisted with the shielding performance test. H.Z. and P.Y. performed the XRD experiment. L.J. and Q.S. fabricated the i-Sleeve and i-Glove. Z. L. and S.Q.X. performed the CNN. L.J. drafted the manuscript, Y.L., K.J.K., S.Q.X, Z.L., and B.R. revised the manuscript, and all authors discussed the results.

\section{Competing financial interests}

The authors declare no competing financial interests. 
628 Materials \& Correspondence

629 Correspondence and requests for materials should be addressed to $\mathrm{Yi} \mathrm{Li}$

630 (henry.yili@manchester.ac.uk), Kap Jin Kim (kjkim@khu.ac.kr), and Sheng Q. Xie 631 (S.Q.Xie@leeds.ac.uk).

632 


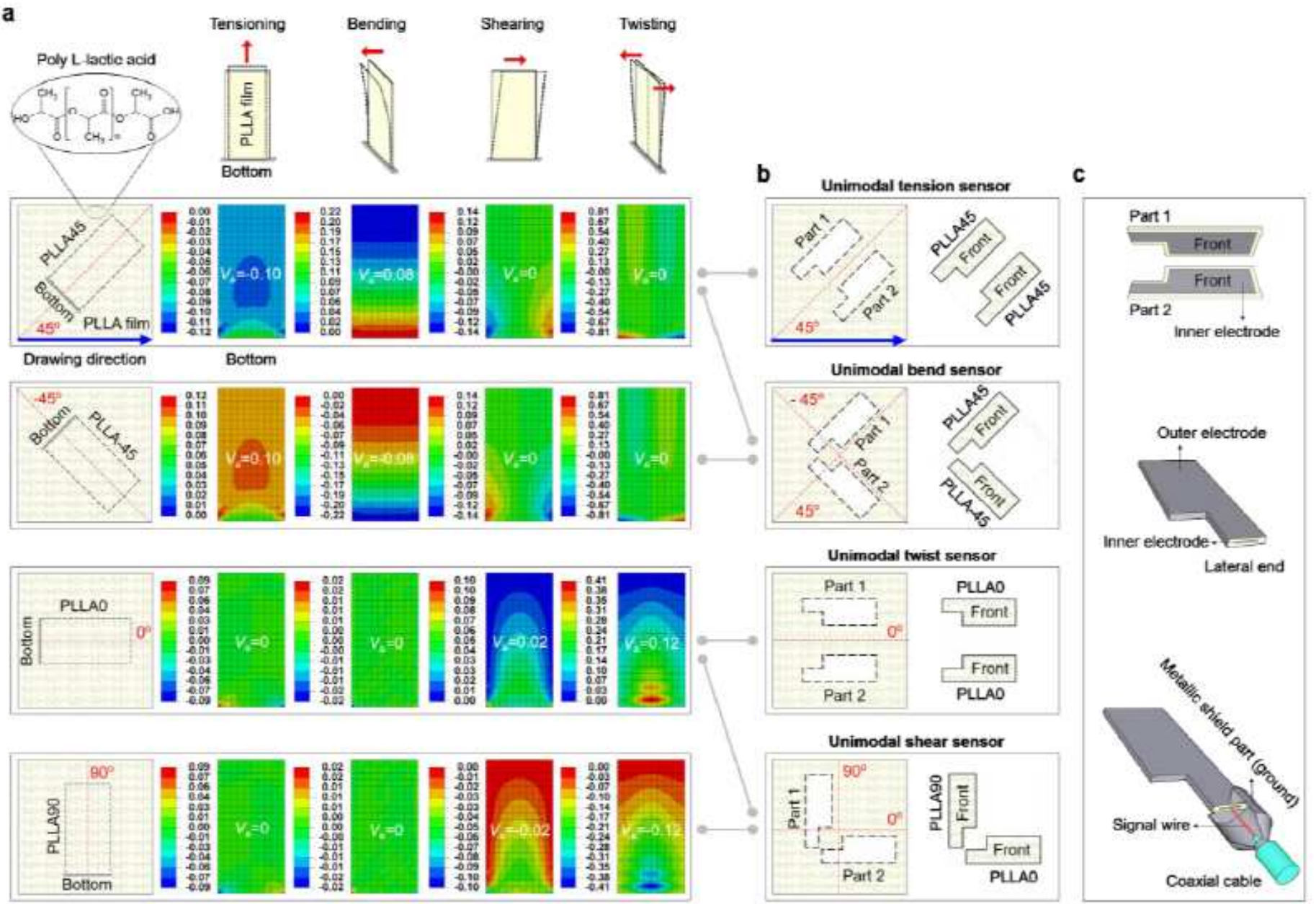

d

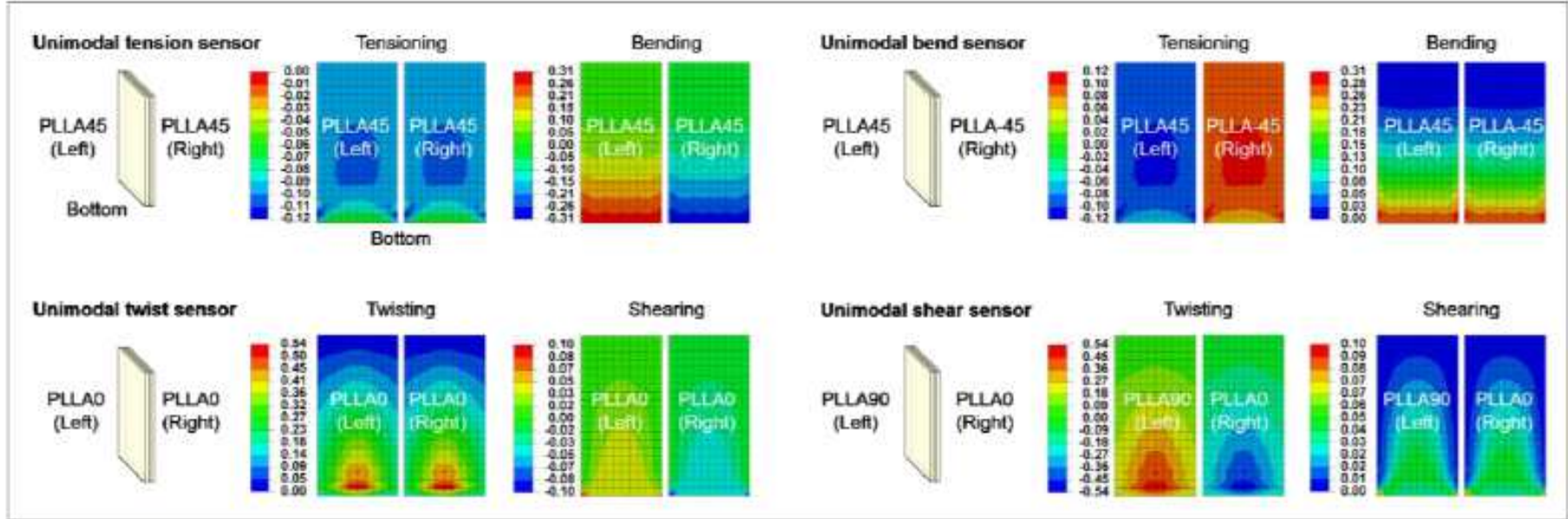

Unit Voit

\section{Figure 1}

Design concept of unimodal tension, bend, twist, and shear sensors. a, Top schematics show the applied forces and their directions (red arrows) for tensioning, bending, shearing, and twisting deformations in finite element simulations. The bottom panels show the piezoelectric response simulations of the 
uniaxially drawn piezoelectric PLLA films with different cutting angles under tensioning, bending, shearing, and twisting deformations. The first panel shows the uniaxially drawn piezoelectric PLLA film with a cutting angle of $45^{\circ}$ (PLLA45 for short) and its cutting schematic (first graph) with a cutting shape (black dash line) and a cutting angle (red dash line), and a PLLA molecular formula shown in the circle. The drawing direction (blue arrow) of the PLLA film is the same as the PLLA molecular chain's direction. The average responding voltage of all nodes of the PLLA film surface $(\mathrm{Va})$ is shown in the middle, and the PLLA film bottom is fixed when it is subject to tensioning, bending, shearing, and twisting forces. The same illustrative schemes are applied for PLLA-45, PLLA0, and PLLA90. b, Left schematics show the cutting angles (red dash lines) and cutting shapes (black dash line) of two parts from an original piezoelectric PLLA film for the unimodal tension, bend, twist, and shear sensors, respectively, and the right schematics show the corresponding parts after cutting out; $c$, Top schematic shows the patterns of inner electrodes (gray areas), and the front sides (i.e., inner electrodes) of two parts of each sensor are stuck together; the middle one shows the exposed area of each assembled sensor is coated with silver paste as an outer electrode, except the lateral end of the sensor; the bottom one shows the inner electrodes are connected to a signal wire, and the outer electrode is connected to a metallic shield part (ground) of a coaxial cable. d, Piezoelectric response simulations of two PLLA films composing each unimodal sensor under tensioning, bending, shearing, and twisting deformations to prove the unimodal sensors' hypothesis. The first panel shows the piezoelectric response of two PLLA45s of the unimodal tension sensor under tensioning and bending. The piezoelectric responses of the exposed areas of two PLLA45s are shown in simulation; in other words, the displayed voltages are on outer electrodes of the unimodal tension sensor. The generated voltages of the two PLLA45s are superimposed when tensioned, but they are offset under bending. The unimodal tension sensor under twisting and shearing is not simulated because the PLLA45 is insensitive to the two deformations, as demonstrated in Fig. 1a. The same illustrative schemes are applied for the unimodal bend sensor under tensioning and bending, the unimodal twist sensor under twisting and shearing, and the unimodal shear sensor under twisting and shearing. Note that all parameters and applied forces used in these simulations (Fig. 1d) are the same as the previous piezoelectric PLLA film simulations (Fig. 1a). 

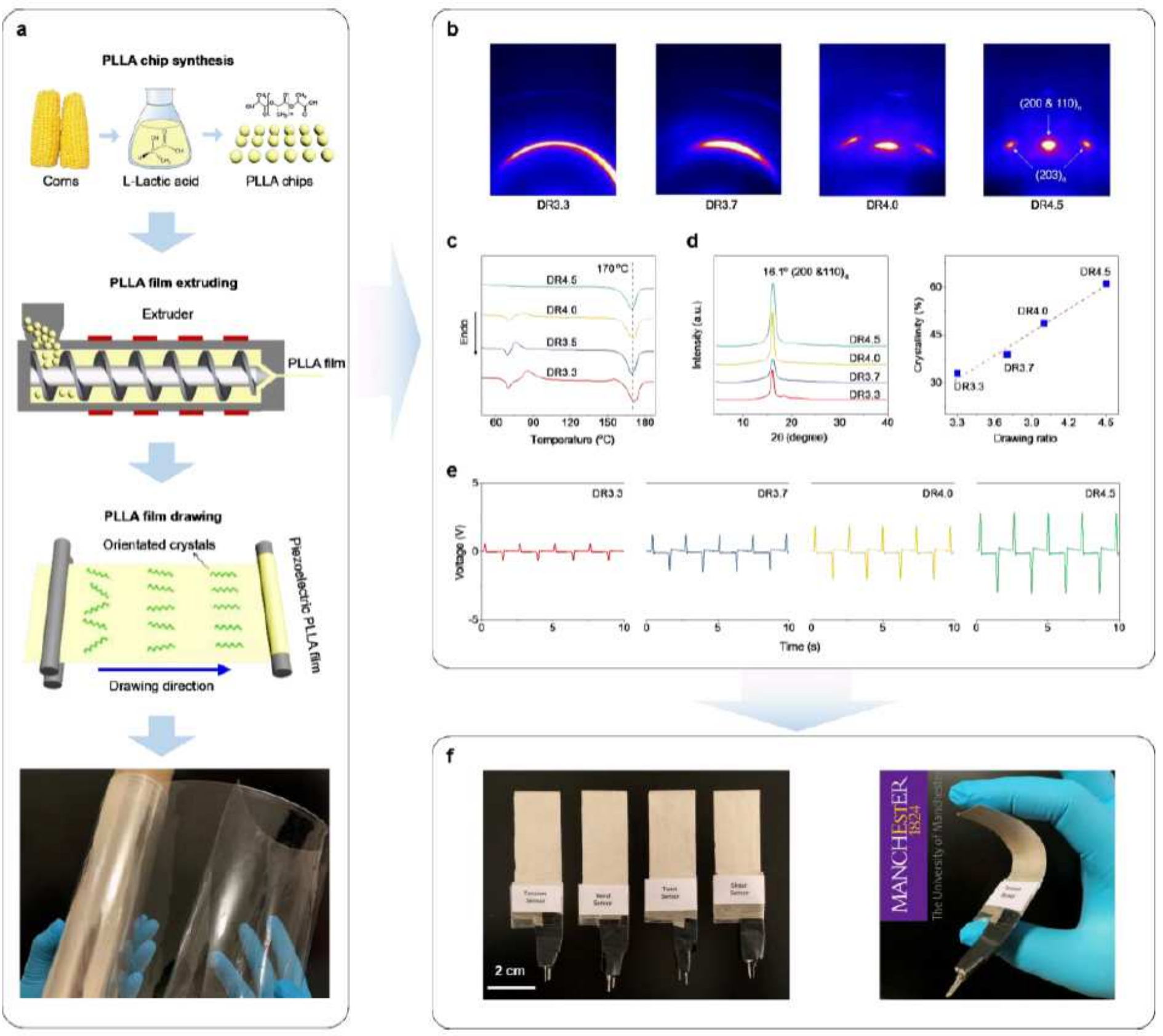

\section{Figure 2}

Preparation and characterization of uniaxially drawn piezoelectric PLLA films. a, Schematic of uniaxially drawn piezoelectric PLLA films preparation process. The first photograph shows the PLLA chip synthesis, which is polymerized from L-lactic acid that is obtained from the fermentation of renewable and biodegradable plant sources such as corns37. In this work, we directly purchased the PLLA chips from NatureWorks, USA. The second photograph shows the PLLA film extruding. The PLLA chips are extruded into PLLA film at $225^{\circ} \mathrm{C}$ using an extruder after dehumidifying at $120^{\circ} \mathrm{C}$ under a vacuum for eight hours. The third photograph shows that PLLA film is stretched at different drawing ratios (DR) of 3.3, 3.7, 4.0, and 4.5 at $70{ }^{\circ} \mathrm{C}$, and each sample names DR3.3, DR3.7, DR4.0, and DR4.5, respectively. The bottom photograph shows a transparent piezoelectric PLLA film (DR4.5). b, Two-dimensional wide-angle x-ray 
diffraction (2D-WAXD) photographs of four PLLA film samples for crystal orientation analysis; C, Differential scanning calorimeter (DSC) melting thermograms of the PLLA films with different DRs for crystal form determination. d, 1D-WAXD spectrum of the PLLA films for crystal form determination (left) and their crystallinity (right). e, Comparison of piezoelectric response of four PLLA film samples under tensioning deformation. The dimension (Length $\times$ Width) of each sensor is $4 \times 2 \mathrm{~cm}$, and every PLLA film is cut at $45^{\circ}$ from the drawing direction. $f$, Photographs of four unimodal sensors (left) and the sensor flexibility demonstration (right).

a

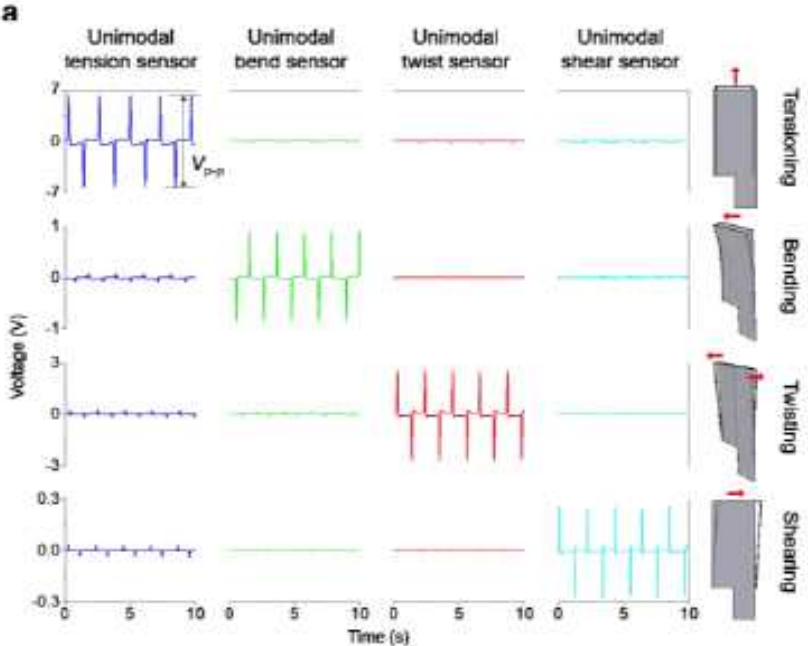

b
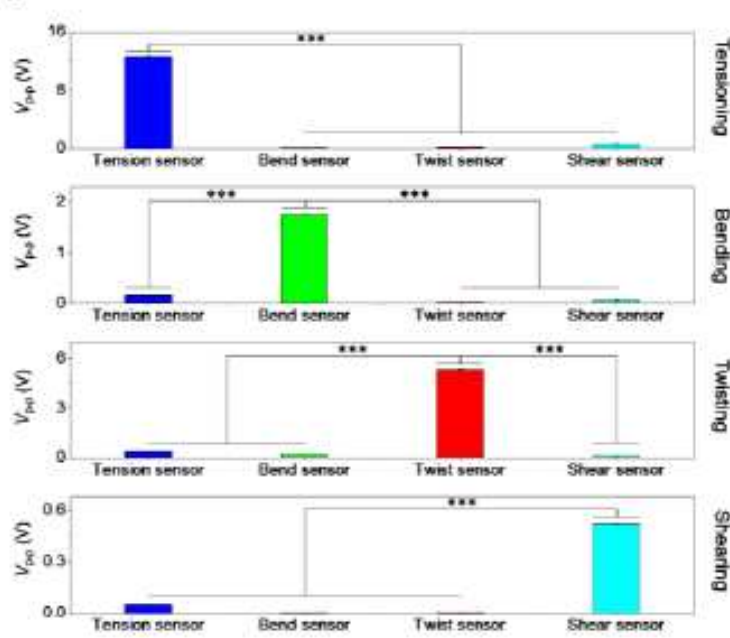

f d
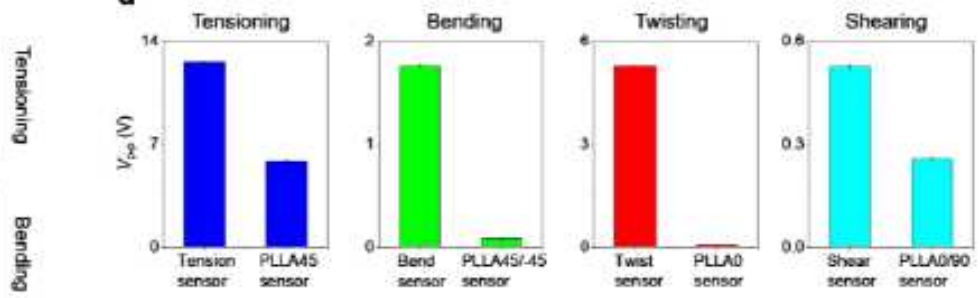

e
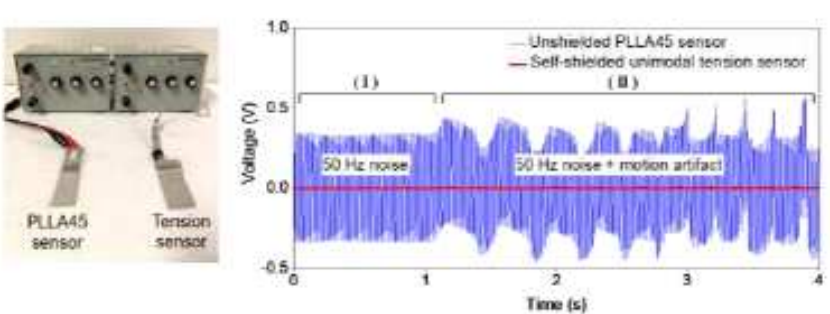

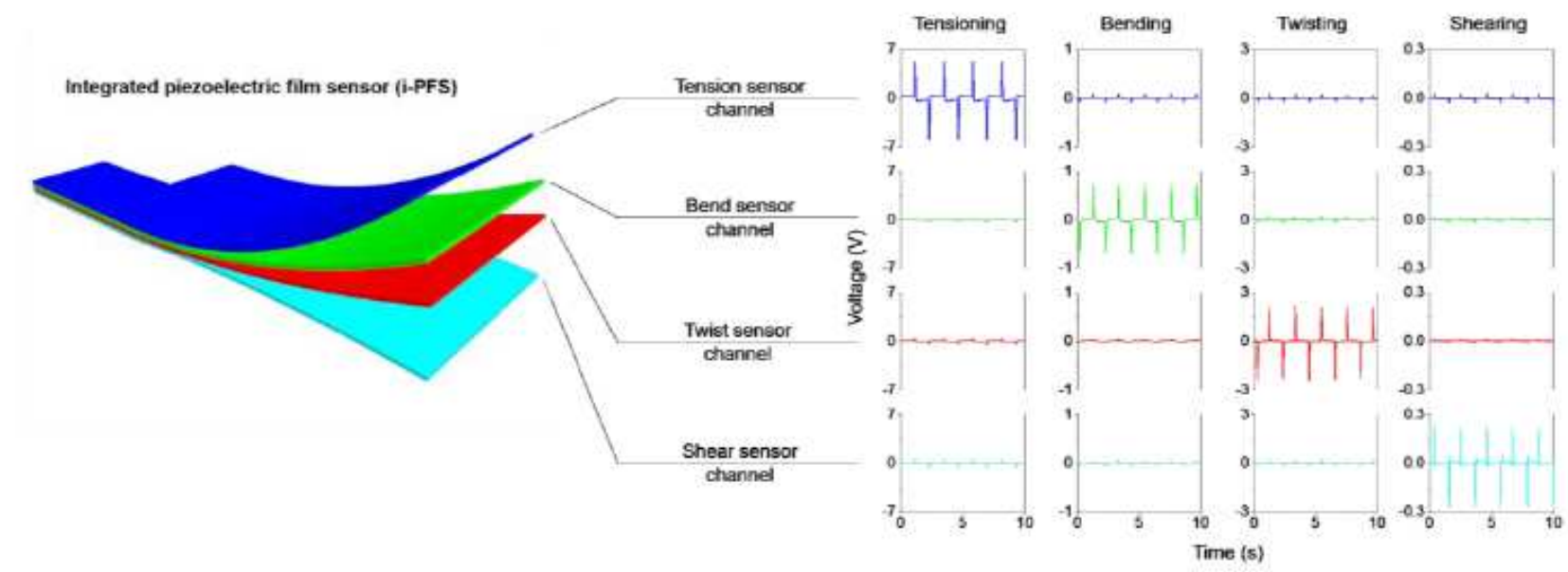




\section{Figure 3}

Performance evaluation of four unimodal sensors and integrated piezoelectric film sensor (i-PFS). a, Piezoelectric responses of each unimodal sensor under the same tensioning, bending, twisting, and shearing conditions. Schematics of unimodal tension, bend, twist, and shear sensors with their corresponding deformations are shown in right insets. $b$, Comparison of the corresponding peak to peak signal amplitudes (Vp-p) of Fig. 3a (*** indicating P-value $<0.001)$. c, Normalized Vp-p of unimodal tension, bend, twist, and shear sensors under tensioning, bending, twisting, and shearing deformation conditions. $d$, Comparison of the piezoelectric response between the four unimodal sensors and the conventional single-layer PLLA sensors under the above conditions. e, The left photograph shows an experimental setup for the shielding performance test. The right figure shows the shielding performance of the unshielded PLLA45 sensor and the self-shielded unimodal tension sensor (I) under the static condition and (II) when walking/running next to two sensors. f, Schematic of the i-PFS comprising unimodal tension, bend, twist, and shear sensors (left). The right panels show the piezoelectric response of the i-PFS under the same tensioning, bending, twisting, and shearing conditions. All conditions are the same as those used for individual unimodal sensor (Fig. 3a). 

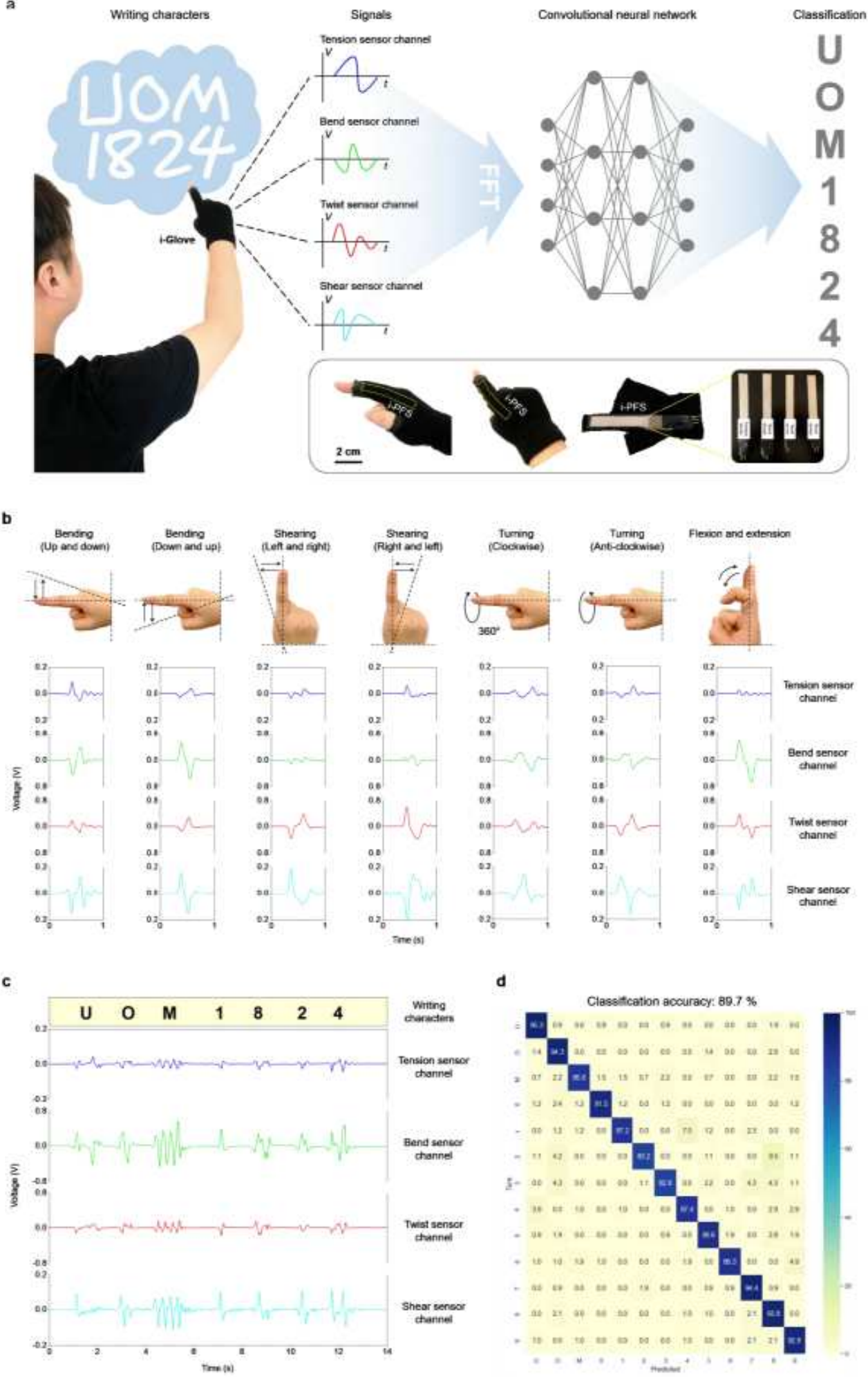

d

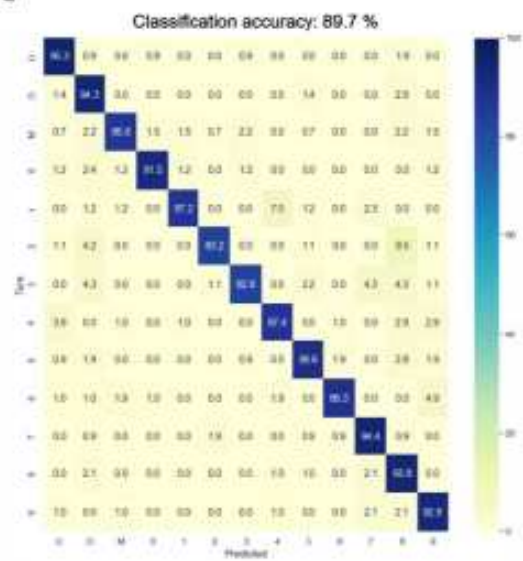

Figure 4

Demonstration of an i-PFS integrated glove (i-Glove) for finger-air-writing application. a, Schematic illustration of a finger-air-writing application. A participant wearing the i-Glove writes some characters, e.g., "U”, "O", “M", "1", "8", "2", “4”, in the free space using his index finger. The four unimodal sensors of the i-PFS collect the corresponding signal of each character. The acquired signals are input into a pretrained convolutional neural network (CNN) program after fast fourier transform (FFT). Finally, the 
program classifies the characters based on the signals. The bottom first two photographs show the i-PFS positions in the i-Glove at the side and top views. The third photograph shows the inside view of the iGlove, in which the i-PFS is inserted into a transparent pocket of the index finger. The last photograph shows four unimodal sensors of the i-PFS. b, Index finger motion classification. Top photographs show index finger motions (bending, shearing, turning, and flexion and extension), and the bottom panels show corresponding raw output voltages from four unimodal sensor channels of the i-PFS. c, Top panel shows writing characters, and the bottom panel shows corresponding raw output voltages of four unimodal sensor channels of the i-PFS. $d$, Confusion matrix for the thirteen characters' classification accuracy and its mean accuracy (top) when using four unimodal sensor channels of the i-PFS.

\section{Supplementary Files}

This is a list of supplementary files associated with this preprint. Click to download.

- Supplementaryinformation.pdf

- Video1forpiezoelectricresponsesimulationAnimationofFig1a.gif

- Video2forshieldingperformancetestunimodaltensionsensorandunshieldedPLLA45sensor.mp4

- Video3foriPFSdemonstration.mp4

- Video4foriSleevedemonstrationwristmotion.mp4

- Video5foriGlovedeomstrationfingerairwriting.mp4 